\title{
Sensorimotor Learning in a Computerized Athletic Training Battery
}

Kristina Krasich, Ben Ramger, Laura Holton, Lingling Wang, Stephen R. Mitroff \& L. Gregory Appelbaum

To cite this article: Kristina Krasich, Ben Ramger, Laura Holton, Lingling Wang, Stephen R. Mitroff \& L. Gregory Appelbaum (2016) Sensorimotor Learning in a Computerized Athletic Training Battery, Journal of Motor Behavior, 48:5, 401-412, DOI: $\underline{10.1080 / 00222895.2015 .1113918}$

To link to this article: http://dx.doi.org/10.1080/00222895.2015.1113918

曲 Published online: 02 Jun 2016.

Submit your article to this journal $\pi$

Џ Article views: 30

Q View related articles $\sqsubset$

View Crossmark data $\asymp$ 
RESEARCH ARTICLE

Sensorimotor Learning in a Computerized Athletic Training Battery

\author{
Kristina Krasich ${ }^{1}$, Ben Ramger ${ }^{1}$, Laura Holton ${ }^{1}$, Lingling Wang ${ }^{1,2}$, Stephen R. Mitroff ${ }^{2,3}$, \\ L. Gregory Appelbaum ${ }^{1,2}$ \\ ${ }^{1}$ Department of Psychiatry and Behavioral Science, Duke University Hospital, Durham, North Carolina. ${ }^{2}$ Center for Cognitive \\ Neuroscience, Duke University, Durham, North Carolina. ${ }^{3}$ Department of Psychology \& Neuroscience, Duke University, \\ Durham, North Carolina.
}

\begin{abstract}
Sensorimotor abilities are crucial for performance in athletic, military, and other occupational activities, and there is great interest in understanding learning in these skills. Here, behavioral performance was measured over three days as twentyseven participants practiced multiple sessions on the Nike SPARQ Sensory Station (Nike, Inc., Beaverton, Oregon), a computerized visual and motor assessment battery. Wrist-worn actigraphy was recorded to monitor sleep-wake cycles. Significant learning was observed in tasks with high visuomotor control demands but not in tasks of visual sensitivity. Learning was primarily linear, with up to $60 \%$ improvement, but did not relate to sleep quality in this normal-sleeping population. These results demonstrate differences in the rate and capacity for learning across perceptual and motor domains, indicating potential targets for sensorimotor training interventions.
\end{abstract}

Keywords: motor control, perception and action, sensorimotor learning, sleep, vision

W hether playing basketball, driving a car, or walking across a city street, success in a number of human endeavors requires individuals to constantly engage with their visual environments. By processing relevant visual information and executing accurate motor responses, sensorimotor skills permit us to effectively detect environmental stimuli, direct our attention to the most relevant information, and orient our bodies to successfully react to the dynamic environment around us. Not only are sensorimotor skills important in our daily activities, they are also associated with achievement in a number of human pursuits. For example, two recent meta-analyses of the sports expertise literature have shown that many, but not all, sensorimotor abilities are superior in more accomplished athletes relative to less accomplished athletes (Mann, Williams, Ward, \& Janelle, 2007; Voss, Kramer, Basak, Parkash, \& Roberts, 2010). Similarly, research has shown that these skills predict success in a number of occupations, such as surgery (Datta, Chang, Mackay, \& Darzi, 2002; Maan, Maan, Darzi, \& Aggarwal, 2012) and military pilot performance (King et al., 2013). In fact, military selection has historically used perceptual and sensorimotor assessments as criteria for screening pilots (Griffin \& Koonce, 1996). Collectively, these studies emphasize the need for proficient sensorimotor skills to attain the highest levels of success.

While important in a myriad of contexts, sensorimotor abilities can also be improved through practice, and there are now numerous examples wherein deliberate practice has resulted in learning that persists for weeks or months (Green \& Bavelier, 2008). For example, through different types of generalized training, individuals are able to improve visual skills, such as visual acuity (Deveau, Lovcik, \& Seitz, 2014; Poggio, Fahle, \& Edelman, 1992; Saarinen \& Levi, 1995), contrast sensitivity (Li, Polat, Makous, \& Bavelier, 2009; Zhou et al., 2006), spatial orientation sensitivity (Shiu \& Pashler, 1992; Vogels \& Orban, 1985), and motion sensitivity (Appelbaum, Schroeder, Cain, \& Mitroff, 2011; Ball \& Sekuler, 1982). This kind of vision training is suggested to improve performance, such as in athletics (Ciuffreda $\&$ Wang, 2004). When considered in the context of motor (Karni et al., 1998) and cognitive (Willis et al., 2006) skills, where learning is frequently reported, there has been an increasing realization that the low-level visual skills that were once considered inelastic can be improved through deliberate practice.

Given the importance of sensorimotor skills, and the literature demonstrating their malleability, there is a growing movement towards developing sports vision training tools to measure and enhance these skills (Erickson, 2007). One such tool is the Nike Sensory Station (Nike, Inc., Beaverton, OR)—a computerized assessment device equipped with a battery of psychometric tasks that are administered with video instructions in about $30 \mathrm{~min}$ by certified trainers. This interactive task battery includes nine measures that have been identified as important abilities for sports performance (Erickson et al., 2011; Hitzeman \& Beckerman, 1993). Specifically, the battery assesses visual skills such as static visual acuity, contrast sensitivity, and depth perception, as well as skills that rely on the coordination of ocular and motor abilities such as near-far quickness (NFQ), dynamic visual acuity (DVA), perception span (PS), eye-hand coordination (EHC), go-no go (GNG), and response times (RSP). The Sensory Stations are deployed in a number of athletic and military training facilities and thus offer a broad platform to study the variability and malleability of sensorimotor skills in important applied contexts.

Correspondence address: L. Gregory Appelbaum, 400 Trent Dr., Durham, NC 27710, USA.e-mail: greg@duke.edu 
Previous research with the Sensory Stations has demonstrated that certain tasks in the battery provide reliable (Erickson et al., 2011; Gilrein, 2014) and cross-validated (Wang et al., 2015) measures that can be used to investigate sensorimotor abilities in relation to performance in realworld endeavors. For example, using logistic regression techniques it was shown that better performance on DVA and visuomotor control measured by the Sensory Stations accounted for nearly $70 \%$ of the variability in goals scored over two seasons in a sample of collegiate hockey players (Poltavski \& Biberdorff, 2014). Additionally, worse overall performance scores on the Sensory Station were associated with an increased likelihood of sustaining head impacts during practices and games for American collegiate football players (Harpham, Mihalik, Littleton, Frank, \& Guskiewicz, 2014), indicating a link between collision avoidance and visuomotor skills. Together, these studies suggest that the sensorimotor skills measured by the Sensory Stations may be directly related to athletic performance and that training programs that targets these skills may lead to more optimal performance outcomes.

Based on preliminary evidence indicating test-retest improvements on the motor-dependent tasks of the Sensory Station battery (Erickson et al., 2011; Gilrein, 2014), in the present study we aimed to more fully quantify the rate and time course of learning that might accompany practice on these tasks. Further, because there are discrepant findings on whether sleep plays a role in enhancing perceptualmotor abilities (Frank \& Benington, 2006; Walker \& Stickgold, 2006) or protecting learned skills from being forgotten (Rickard, Cai, Rieth, Jones, \& Ard, 2008), measures of sleep quality were also assessed via actigraphy and selfreported sleep logs for each individual throughout the duration of this study. By quantifying learning in these important visual and motor skills, the present study has the capacity to inform both theory regarding behavioral and brain plasticity that results from practice and the applied use of sensorimotor training interventions to improve realworld athletic and occupational outcomes. In particular, the present study may suggest specific abilities to target in athletic training activities and specific training sequences that would lead to expected improvements.

\section{Method}

\section{Participants}

Twenty-seven individuals (14 women) between the ages of 18 and 28 years old $(M$ age $=22.04$ years, $S D=$ 2.65 years) participated in a four-day experimental protocol approved by the Duke University Institutional Review Board. Participants were compensated \$20/hour for their efforts, and data from the first assessment session performed on the Nike Sensory Station is also included in a separate report on the state influences of sensorimotor control in Wang et al. (2015).

\section{Procedure}

The experimental protocol consisted of four consecutive days of contact with the participants. On the first day, participants were given study instructions and signed informed consent. In addition, they were given actigraphy equipment and a written sleep log for monitoring their sleep cycles over the remainder of the study duration (more details subsequently).

On the second day of the protocol, participants returned to the laboratory and were administered an initial assessment on the Sensory Station battery, which included a onetime entering of profile information, full audio-visual instructions and a brief practice for each task (more details subsequently). After completing this first session on the Sensory Station participants then performed two more sessions that included practice trials before each task, but did not include audio-video instructions.

On the third day, participants completed four sessions of the Sensory Station battery. These sessions did not include audio-video instructions, however, participants were given a brief practice trial before each task to become refamiliarized with the instructions, and participants were encouraged to ask the experimenter questions if further clarity was required.

On the fourth and last day of study, participants performed three sessions on the Sensory Stations. The last of these included task instructions in order to mirror the initial session of the first day as closely as possible. In whole, participants completed ten sessions, each lasting about 20$25 \mathrm{~min}$, over the course of days $2-4$.

\section{Measures and Tasks}

\section{Sensory Station}

Behavioral assessments were performed on the Nike Sensory Station, a computerized device consisting of nine sports-relevant psychometric tasks that measure various visuoperceptual and visuomotor abilities. This device is equipped with two high-resolution LCD monitors: one 23inch, $120-\mathrm{Hz}$ display and one 42-inch, 60-Hz touch-sensitive display, both controlled by a single computer. Custom software controls the stimuli display, response collection, and test procedures based on participants' responses. Prerecorded video instructions are automatically played at the start of each task. Brief descriptions for each task are included below and schematic illustrations are displayed in Figure 1. More detailed reports of task procedures are included in (Erickson et al., 2011; Poltavski \& Biberdorff, 2014). Additional self-reported information about the participant's age, gender, height, eye correction, and sports activities was collected, but not used in these analyses as they did not relate to the primary research question of sensorimotor learning. Further, self-reported information about each participant's concussion history was registered, but 


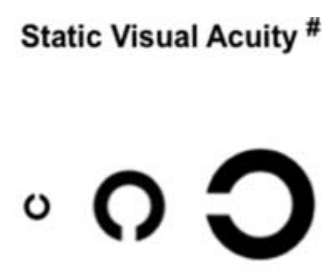

Dynamic Visual Acuity \#

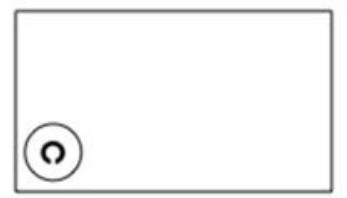

Eye-Hand Coordination

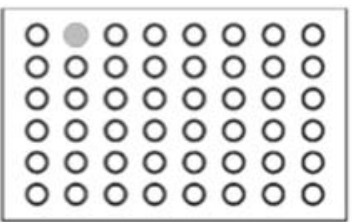

Contrast Sensitivity \#

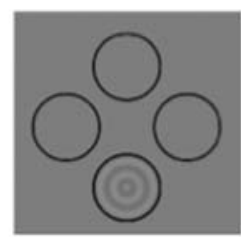

Near-Far Quickness

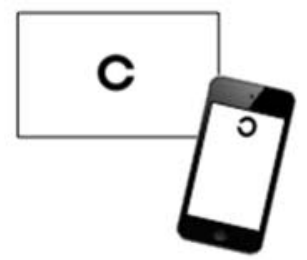

Go/No Go

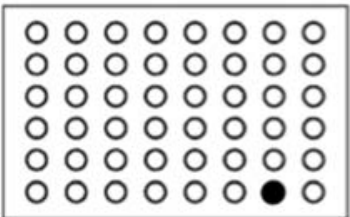

Depth Perception \#

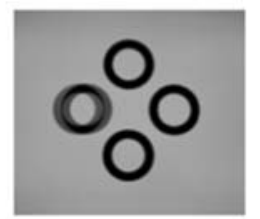

Perception Span

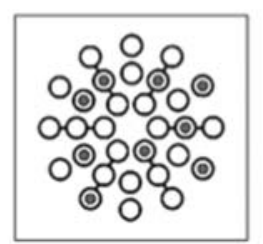

Hand Response Time

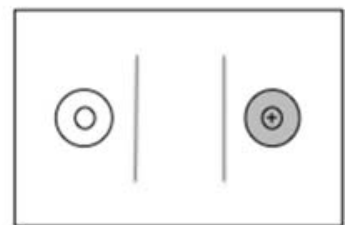

FIGURE 1. Illustrations of the nine perceptual and visuomotor tasks included in the Nike SPARQ Sensory Station battery. \# indicates tasks that performed under a staircase schedule.

was not analyzed because only three individuals reported ever experiencing concussions and were all greater than one year prior to the assessment.

\section{Staircase Visual Sensitivity Tasks}

The Sensory Station battery consists of four tasks that measure visual sensitivity thresholds and five that assess visuomotor abilities. The four tasks measuring visual sensitivity thresholds - static visual acuity (SVA), contrast sensitivity (CS), depth perception (DP), and DVA-were performed on a 23-inch, high-resolution display monitor with participants standing $16 \mathrm{ft} \int(4.9 \mathrm{~m})$ away from the Station and responding with a handheld Apple iPod Touch (Apple Inc., Cupertino, CA) wirelessly connected to the Station computer. Responses were made with one-direction finger swipes on the iPod screen. Stimuli presentation and final thresholds were determined on a staircase procedure wherein the initial stimuli were displayed at a preset threshold value and were dynamically adjusted according to the performance of the participant. With each correct response, the following stimulus was presented at a more difficult level, while an easier level followed incorrect responses. The staircase ended when two adjacent levels of correct and incorrect responses were recorded; the hardest level with two correct responses determined the threshold.
The SVA task assessed the minimum detectable spatial resolution for a nonmoving, black Landolt ring presented on a white background. The gap of the ring was either at the top, bottom, left, or right of the ring, and participants were asked to swipe the touch screen in the direction of the gap. The initial resolution was set equivalent to $20 / 50$ Snellen acuity, and adjusted following the staircase procedures described above (range $=4-18$ steps; average $=$ 10.4). The stimulus stayed on the screen until a response was given. Right monocular, left monocular and binocular acuities were measured in LogMar units and averaged for analysis purpose, with smaller values indicating better performance. In past research, the SVA task has been crossvalidated using a Snellen Eye Chart and was shown to be a valid measure of visual acuity (Wang et al., 2015).

The CS task assessed the minimum contrast level to distinguish lightness and darkness on a grey background. Participants were presented four circles in a diamond formation and asked to identify which circle contained a pattern of concentric rings by swiping in the direction of the circle. Stimuli were adjusted according to the staircase procedure (range $=3-17$ steps; average $=9.6$ ) and remained on the screen until a response was given. Final contrast thresholds were measured between $10 \%$ and $1.0 \%$ contrast at six cycles per degree (cpd), and between $32 \%$ and $2.5 \%$ contrast at $18 \mathrm{cpd}$. Measurements were log transformed and averaged across the two spatial frequencies 
with larger values indicating better performance. Previous research has shown that performance on the CS task is highly correlated to other measures of contrast sensitivity (Lesmes, Lu, Baek, \& Albright, 2010), indicating that the CS task is a valid measurement (Wang et al., 2015).

The DP task measured the threshold at which the smallest amount of disparity required to identify differences in depth. Because stereoacuity differs for different head positions (Yoo, Reichow, \& Erickson, 2011), DP included three viewing conditions: with participants directly facing the display, turned to the right and looking at the display over their left shoulder, and turned to the left and looking at the display over their right shoulder. Participants wore a pair of liquid crystal goggles (NVIDIA 3D Vision, Santa Clara, CA) connected via wireless link to the Station computer. The liquid crystal shutter system created simulated depth in one of four black rings presented on a light gray background and participants were asked to identify, by swiping on the iPod, which ring appeared to have depth. Stimuli stayed on the screen until a response was given, and depth perception thresholds were determined with the staircase procedure (range $=3-21$ steps; average $=9.1$ ) and measured as the smallest amount of disparity in arc seconds required for the ring with depth to be identified. The average threshold for the three conditions was used for analysis, with smaller average thresholds indicating better performance.

DVA tested the ability to resolve briefly presented peripheral targets by measuring the minimal amount of time needed to correctly identify the gap direction of a slightly suprathreshold Landolt ring. On each trail a Landolt stimuli set at $110 \%$ of SVA was briefly displayed in one of the four corners of the screen, and participants were asked to identify which cardinal direction the gap was facing by swiping the iPod in the corresponding direction. The duration of presentation of the Landolt ring started at $250 \mathrm{~ms}$ and was dynamically adjusted according to the staircase procedure (range $=5-36$ steps; average $=12.4$ ). Final dynamic visual acuity scores were the minimum amount of time in milliseconds required to correctly identify the gap in the ring, with shorter durations indicating better performances.

\section{Psychomotor Tasks}

Five of the tasks performed on the Sensory Station assessed visual abilities in coordination with motor skills. These tasks measured how quickly and accurately a stimulus could be perceived, processed and engaged. During one of the tasks (NFQ), participants stood $16 \mathrm{ft}$ away and responded using the iPod touch. For the other four tasks, participants stood at arm length and used the 42-inch touchsensitive screen positioned at eye level to respond. These tasks did not use a staircase procedure.

The NFQ measured how quickly participants could visually accommodate between near and far targets. During this task, participants stood 16 feet away from the Station and held up the iPod at arm's length so that the top of the handheld screen appeared to align with the bottom of the 23-inch display on the Station. A black Landolt ring was alternatingly presented at the top of the near screen of the iPod and the center of the far screen of the Station. Participants were instructed to swipe the iPod screen to indicate the direction of the gap as quickly as possible. The next stimulus appeared on the successive screen only after a correct response was registered for the current stimulus. Scores were the total number of correct responses made in 30 seconds.

The PS task tested spatial working memory for briefly presented patterns of dots. During this task, a grid of circles in a radial configuration was displayed for $100 \mathrm{~ms}$ on the large 42-inch touch screen. For each trial a pseudorandomized pattern of green dots flashed briefly within a subset of the circles and participants were instructed to recreate the pattern of the dots by touching the corresponding circles. As participants progressed through the task, the size of the grid and the number of dots presented increased. The task ended when participants could no longer reach a passing score $(100 \%$ correct for the first three levels and $75 \%$ correct for the higher levels) on two successive trials for a given level (range $=4-14$ levels; average $=10.1$ ). PS scores were computed as the total number of correctly identified dots minus the number of missed or falsely identified dots across all of the trials. Across the whole sample, $88 \%$ of possible targets were correctly identified, $12 \%$ were missed, and 5.6 false alarms were made per individual.

EHC measured the ability to quickly and accurately touch a sequence of briefly presented targets. During the task a green dot would appear pseudorandomly on a display in one of 48 locations on the screen (in a $8 \times 6$ grid), and participants were to touch the green dot as rapidly as possible using either hand. Once the target was touched, auditory feedback was given, the dot would disappear and a subsequent green dot would immediately appear in another location on the grid. This would continue until a sequence of 96 dots was successfully completed (two at each of the 48 locations). The score for EHC was the total time it took to touch all 96 dots in the sequence.

GNG was a response inhibition task that tested the ability to respond to go targets while not responding to no-go nontargets. This task was similar to EHC except both green and red dots would appear. Participants were instructed to touch the green dots but to withhold responses to the red dots. In this task dots would only appear for $500 \mathrm{~ms}$ before a subsequent dot would appear and this happened until 96 total dots (64 green, 32 red) cycled through the task. Auditory feedback was provided for both hits (high tone) and false alarms (low tone) and final GNG scores were computed as the total number of targets successfully engaged minus the number of red dots incorrectly touched.

The RSP task measures simple motor reaction time in response to a visual stimulus. During this task two rings 
were presented on either side of the 42-inch touch screen. Participants were asked to place the fingertips of their dominant hand in the starting ring on that side of their body while aligning their body with the other landing ring. When the landing ring turned green, the participant disengaged from the starting ring and made a ballistic hand movement to touch the landing ring as quickly and accurately as possible. Participants would complete seven trials, with the possibility to repeat up to two of these trials if they were slower than two standard deviations from the mean. RSP was computed as the average time it took to disengage from the starting ring and engage with the landing ring for the seven trials.

\section{Sleep Measures}

Previous studies have shown performance improvements in subsequent testing for both perceptual discrimination (Karni, Tanne, Rubenstein, Askenasy, \& Sagi, 1994) and motor tasks (Walker, Brakefield, Morgan, Hobson, \& Stickgold, 2002) following a night of sleep when compared to equally elapsed periods of wakefulness. Other studies, however, have not observed interactions between sleep and perceptual learning (Aberg, Tartaglia, \& Herzog, 2009; Hussain, Sekuler, \& Bennett, 2009), leaving the role of sleep in learning an open question. In light of these findings, we recorded actigraphic and self-reported measures of sleep throughout the study period to assess the relationship between sleep and sensorimotor performance on the Sensory Station tasks. Actigraphy data was collected in 15-s epoch lengths using the Actiwatch 2 (Actiwatch 2, Philips Respironics, Andover, MA). Self-report measures of sleep activities were recorded on a written sleep log that was completed by participants each evening before bed and morning upon awakening. The sleep log was modeled after The Consensus Sleep Diary (Carney et al., 2012), and participants described characteristics of their previous night of sleep, such as estimated sleep onset and offset times, number and duration of any nighttime awakenings, and restfulness of the sleep. Answers from the sleep log were used as a guide to confirm rest intervals during actigraphy analysis.

\section{Analyses}

\section{Behavioral Measures}

Of the 27 individuals who participated, 26 successfully completed all 10 sessions of the Sensory Station. One participant performed only two sessions on the final day, completing only nine total sessions over the four days, with the final session including full instructions. For analysis purposes, scores from this participant's ninth session were duplicated as their scores for the tenth session.

To test the hypothesis that behavioral performance exhibited significant change over the ten practice sessions, linear and quadratic coefficients for change were first calculated for each of the nine tasks over the ten practice sessions. One-way $t$ tests were performed to assess whether the resulting linear and quadratic coefficients were significantly different from zero. As such, these results indicated whether there was a change in performance over the 10 training sessions in each of the tasks, as well as any variation in the rate of change over sessions.

Separate follow-up analyses were performed to more specifically describe learning in some of the tasks that showed improvements in the analyses described previously. Within the NFQ task, response times were summed individually for the responses to stimuli presented on the near display (near) and for the responses to the stimuli presented on the far display (far). Linear and quadratic coefficients were calculated separately for near and far trials and one-sample $t$ tests were performed to test for selective changes in performance over the ten sessions.

The RSP task was subdivided into reaction times, the average time in milliseconds it takes to disengage ones hands from the starting ring after the target is displayed, and rotor times, the average time it takes to reengage with the target ring. Linear and quadratic coefficients were calculated for these and one-sample $t$ tests were conducted to assess whether changes over the course of the ten sessions were significantly different from zero.

EHC and GNG were both performed with stimuli presented on a virtual $8 \times 6$ grid, spanning the extent of the 42-inch touch screen display. To assess how learning manifest over the spatial extent of this display the grid was subdivided into three regions - the center, an inner ring, and an outer ring (see Figure 3 ) - and a $3 \times 2$ analysis of variance (ANOVA) comparing the three regions and the two sessions was conducted for both tasks. ANOVAs were corrected according to the Greenhouse-Geisser criteria.

\section{Sleep Measures}

Actigraphy was recorded continuously on each participant over the duration of the four-day experiment and rest intervals were manually set with guidance from motion activity, ambient light, and self-report measurements. Average sleep duration and average sleep efficiency-which is defined as the ratio of time asleep to time spent attempting to sleep-were calculated using the proprietary algorithm within the Actiwatch commercial software. Due to a miscalibration of the Actiwatch, one participant did not have actigraphy data and was removed from analyses involving sleep. Pearson's correlations were computed to assess whether these sleep measures were related to task performance or learning coefficients. In addition to assessing how individual participant sleep metrics impacted psychometric performance, we also compared whether or not performance differed between sessions that occurred successively within the same day versus changes that occurred between sessions that were separated with a night of sleep. 


\section{Results}

\section{Performance Summaries}

Behavioral performance on the nine Sensory Station tasks was in close agreement with previously reported performance for other samples of healthy, college-aged individuals (Erickson et al., 2011; Gilrein, 2014; Wang et al., 2015). Means for the nine tasks, across the 10 sessions, are shown in Table 1. Across the twenty-seven participants, performance adhered to normal distributions for the NFQ, DVA, PS, EHC, GNG, and RSP tasks (all Shapiro-Wilk $p$ s $<.03$ ). Performance did not adhere to normal distributions for the SVA, CS, and DPT tasks ( $p$ s $>.45$ ), as these tasks produced a limited number of threshold values under the staircase schedule used.

\section{Sensorimotor Learning}

Our primary goal in the present study was to characterize and compare the rate of learning that occurred over repeated practice with the nine sensorimotor tasks. Toward this aim we observed significant improvements in performance in five of the nine tasks (see Figure 2 and Table 2). Specifically, linear coefficients were significantly different from zero for the NFQ, PS, EHC, GNG, and RSP tasks. In each of these five cases, the linear change indicated improved performance over the course of the 10 sessions, with improvements of $46 \%$ for NFQ, $25 \%$ for PS, $16 \%$ for EHC, $61 \%$ for GNG, and 9\% for RSP, relative to performance on the first session. Linear coefficients did not differ significantly from zero for SVA, CS, DP, and DVA.

Quadratic coefficients indicate a change in the rate of learning. Among the nine tasks, quadratic coefficients differed significantly from zero for only the EHC and GNG tasks. The values of these coefficients (positive for EHC and negative for GNG) indicate decelerating rate of improvement such that practice effects were larger in early sessions but were reduced in later sessions.

\section{Component Learning for Individual Tasks}

As described previously, significant improvements in performance were seen on five of the nine tested tasks. To shed light on what specific aspects of each task improved, we further analyzed different subcomponents of these tasks.

NFQ requires rapid changes in visual convergence of the eyes to near targets, as well as divergence to far targets. The observed learning in this task may therefore stem from improvements in either, or both, of these types of eye movements. To investigate this further, coefficients of change were calculated separately for response times to near and far trials. One-sample t-tests (Table 3) revealed that both near and far trials showed significant linear improvements throughout the ten sessions $(p<.001)$, with slightly greater improvement in reaction times for near trials (34\% improvement) relative to far trials $(30 \%$ improvement).

The RSP task used in this experiment measures motor actions in response to visual stimuli, such that participants must quickly detect a go signal and disengage their hand from the starting ring (reaction time), and then make a ballistic hand movement to touch the landing ring on the touch-screen monitor (motor time). Enhancements in either of these two subcomponents of the task could be driving the overall performance improvements throughout the ten sessions. To investigate this, coefficients for change were calculated for reaction times and motor times and submitted to onesample $t$ tests. Results (Table 3) indicated that both

TABLE 1. Average behavioral performance and standard deviations (in parentheses) for each task across the ten sessions

\begin{tabular}{|c|c|c|c|c|c|c|c|c|c|c|c|c|c|c|c|c|c|c|}
\hline & \multicolumn{2}{|c|}{$\begin{array}{l}\text { SVA } \\
\log M A R\end{array}$} & \multicolumn{2}{|c|}{ CS $\log$} & \multicolumn{2}{|c|}{$\begin{array}{l}\mathrm{DP} \\
\operatorname{arcsec}\end{array}$} & \multicolumn{2}{|c|}{ DVA $m s$} & \multicolumn{2}{|c|}{ NFQ sum } & \multicolumn{2}{|c|}{ PS score } & \multicolumn{2}{|c|}{$\begin{array}{l}\text { EHC } \\
\text { seconds }\end{array}$} & \multicolumn{2}{|c|}{$\begin{array}{l}\text { GNG } \\
\text { score }\end{array}$} & \multicolumn{2}{|c|}{$\operatorname{RSP} m s$} \\
\hline & $M$ & $S D$ & $M$ & $S D$ & $M$ & $S D$ & $M$ & $S D$ & $M$ & $S D$ & $M$ & $S D$ & $M$ & $S D$ & $M$ & $S D$ & $M$ & $S D$ \\
\hline ession 1 & -0.11 & 0.15 & 1.87 & 0.21 & 66.2 & 59.9 & 283.3 & 125.0 & 23.4 & 5.2 & 38.2 & 11.7 & 53.3 & 2.6 & 24.4 & 10.5 & 493 & 72 \\
\hline & -0.16 & 0.20 & 1.84 & 0.32 & 68.9 & 54.6 & 276.9 & 100.7 & 24.6 & 6.6 & 40.5 & 13.2 & 52.5 & 3.3 & 28.8 & 11.6 & 475 & 54 \\
\hline Sess & -0.12 & 0.11 & 1.92 & 0.21 & 56.3 & 47.9 & 250.0 & 102.6 & 27.6 & 4.3 & 42.6 & 10.6 & 50.3 & 3.7 & 30.3 & 10.9 & 474 & 54 \\
\hline $\operatorname{ssion} 4$ & -0.15 & 0.11 & 1.84 & 0.23 & 62.4 & 53.6 & 281.5 & 146.5 & 28.4 & 5.8 & 43.9 & 10.7 & 50.3 & 3.7 & 31.1 & 10.6 & 477 & 55 \\
\hline & -0.13 & 0.12 & 1.88 & 0.25 & 72.5 & 62.8 & 249.1 & 97.7 & 29. & 7.5 & 44.5 & 11.2 & 49.2 & 3.8 & 32.4 & 11.8 & 451 & 52 \\
\hline es & -0.17 & 0.11 & 1.89 & 0.22 & 71.8 & 56.4 & 299.1 & 146.2 & 28.6 & 6.0 & 41.7 & 9.9 & 48.3 & 4.4 & 34.6 & 10.8 & 453 & 42 \\
\hline & -0.13 & 0.11 & 1.83 & 0.24 & 69.1 & 53.3 & 263.0 & 122.5 & 32.7 & 6.1 & 44.5 & 11.7 & 47.7 & 4.6 & 37.0 & 11.8 & 455 & 50 \\
\hline Ses & -0.14 & 0.12 & 1.88 & 0.20 & 74.9 & 73.3 & 287.0 & 99.4 & 32.7 & 6.3 & 42.3 & 11.4 & 48.2 & 3.9 & 36.3 & 11.2 & 455 & 58 \\
\hline & -0.11 & 0.14 & 1.83 & 0.25 & 78.5 & 67.3 & 275.0 & 115.2 & 33.2 & 6.6 & 44.3 & 12.3 & 47.1 & 4.2 & 38.8 & 11.5 & 453 & 56 \\
\hline on 10 & -0.15 & 0.11 & 1.88 & 0.23 & 75.5 & 64.4 & 274.1 & 107.7 & 34.1 & 6.8 & 47.8 & 11.7 & 46.1 & 4.0 & 39.4 & 10.9 & 451 & 51 \\
\hline Mean & -0.14 & 0.13 & 1.87 & 0.24 & 69.6 & 59.4 & 273.9 & 116.4 & 29.5 & 6.1 & 43.0 & 11.4 & 49.3 & 3.8 & 33.3 & 11.2 & 464 & 54 \\
\hline
\end{tabular}




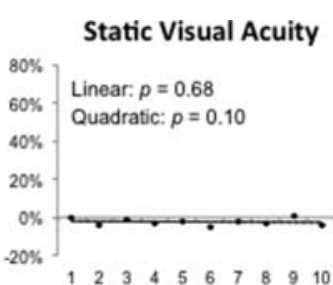

Dynamic Visual Acuity

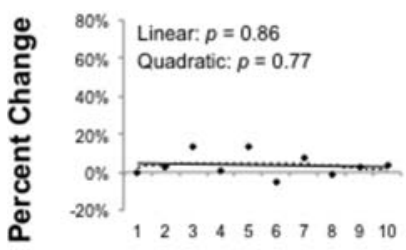

Eye-Hand Coordination

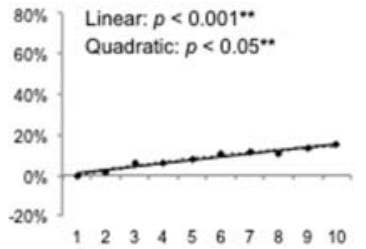

Contrast Sensitivity

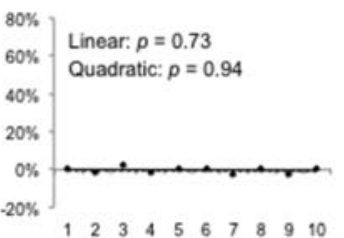

Near-Far Quickness

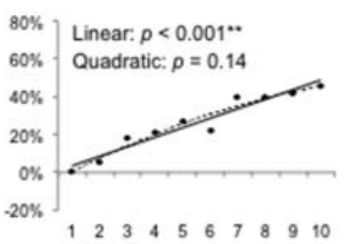

Go / No-Go

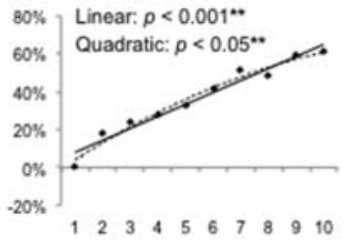

Session

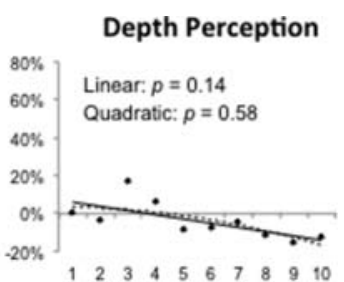

Perception Span

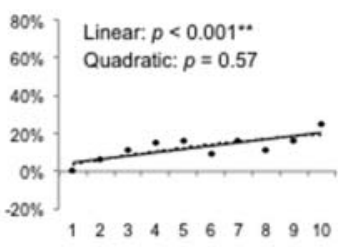

Hand Response Time

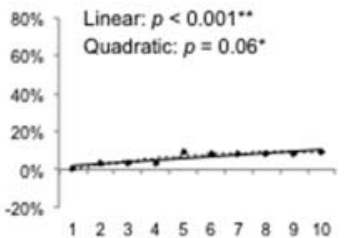

FIGURE 2. Percent change for each task relative to performance on the first session. Solid lines indicate the linear fit for each task. Dashed lines indicate quadratic fits for each task. Significance levels for linear and quadratic coefficients of change are indicated above each subplot. * $p<.05, * * p<.01$.

reaction times and motor times showed significant linear improvements throughout the 10 sessions $(p<.02)$, with greater improvements in motor times $(15 \%$ improvement) relative to reaction times $(6 \%$ improvement).

The EHC and GNG tasks both require quick and dynamic visuomotor coordination that is widely distributed over space on the 42-inch touch-screen monitor. As these two tasks both showed substantial learning over the 10 sessions, we wished to further quantify the spatial extent of improvements driving these enhancements. For this purpose the array was divided into three concentric regions (Figure 3, right) and reaction times were submitted to a $3 \times 2$, region by session ANOVA (shown graphically in Figure 3, left).

TABLE 2. Mean coefficients for each of the nine tasks and results from one-sample t-tests.

\begin{tabular}{|c|c|c|c|c|}
\hline & \multicolumn{2}{|c|}{ Linear coefficients } & \multicolumn{2}{|c|}{ Quadratic coefficients } \\
\hline & $t$ & $p$ & $t$ & $p$ \\
\hline $\operatorname{SVA}(-)$ & -0.42 & .681 & 1.73 & .095 \\
\hline $\mathrm{CS}(+)$ & -0.35 & .726 & -0.07 & .942 \\
\hline $\mathrm{DP}(-)$ & 1.53 & .138 & 0.57 & .576 \\
\hline $\operatorname{DVA}(-)$ & 0.18 & .858 & 0.30 & .768 \\
\hline NFQ $(+)$ & 10.51 & $.001^{* *}$ & -1.54 & .135 \\
\hline PS $(+)$ & 4.00 & $.001^{* *}$ & -0.58 & .565 \\
\hline $\mathrm{EHC}(-)$ & -13.51 & $.001^{* *}$ & 2.34 & $.027^{*}$ \\
\hline GNG $(+)$ & 6.61 & $.001^{* *}$ & -2.34 & $.027^{*}$ \\
\hline $\operatorname{RSP}(-)$ & -4.50 & $.001^{* *}$ & 1.92 & .066 \\
\hline
\end{tabular}

Note. The direction of better performance is shown in parentheses for each task. ${ }^{*} p<.05{ }^{* *} p<.01$. There are 26 degrees of freedom for all t-tests listed. 
TABLE 3. Linear and coefficients and one-sample $t$ tests for measurements that are components of the NFQ and RSP tasks

\begin{tabular}{|c|c|c|c|c|c|c|}
\hline & \multicolumn{3}{|c|}{ Linear coefficients } & \multicolumn{3}{|c|}{ Quadratic coefficients } \\
\hline & $M$ & $t$ & $p$ & $M$ & $t$ & $p$ \\
\hline NFQ-near & -6789.2 & -7.26 & $.001^{* *}$ & * 1259.2 & 2.83 & $.009^{*}$ \\
\hline NFQ-far & -7708.2 & -5.84 & $.001^{* *}$ & * 996.9 & 1.21 & .236 \\
\hline Reaction time & e -229.6 & -2.56 & $.017^{* *}$ & 240.0 & 2.74 & $.011^{*}$ \\
\hline Motor time & -391.1 & -3.67 & $.001^{* *}$ & 32.6 & 0.59 & .561 \\
\hline
\end{tabular}

For the EHC tasks, this ANOVA (Table 4) revealed a main effect of region, $F(1.68,43.79)=91.08, p<.001$, $\eta_{\mathrm{p}}^{2}=0.78$; and a main effect of session, $F(1,26)=$ 234.13, $p<.001, \eta_{\mathrm{p}}^{2}==0.9$; but no region by session interaction, $F(1.94,50.45)=0.74, p=.48, \eta_{\mathrm{p}}^{2}==0.03$. Post hoc paired-sample $t$ tests further determined that changes from session one to session ten were significant in all three regions ( $p<.001$; center $t=8.07$, inner $t=8.10$, outer $t=9.34$ ).

To assess the spatial extent of learning for GNG accuracy was computed at each location based on the number of green 'go' trials in each region. This $3 \times 2$ ANOVA (Table 4) showed a main effect of region, $F(1.78,46.27)=59.41, p<$ $.001, \eta_{\mathrm{p}}^{2}==0.7$; a main effect of session, $F(1,26)=53.34$, $p<.001, \eta_{\mathrm{p}}^{2}==0.67$; and a trending region by session interaction, $F(1.83,47.65), p=.053, \eta_{\mathrm{p}}^{2}==0.11$. Post hoc paired sample $t$ tests further determined that changes in
GNG from sessions 1-10 were significant in all three regions: center $(t=-3.88, p=.001)$, inner $(t=-7.90$, $p<.001)$, and outer rings $(t=-6.17, p<.001)$. Based on these spatial analyses it can be inferred that learning occurred rather uniformly over the spatial extent of the display.

\section{Assessing the Role of Sleep on Sensorimotor Learning in the Sensory Stations}

Because of the discrepant findings on sleep exclusive learning enhancements (Rickard et al., 2008; Walker \& Stickgold, 2006), we also assessed whether sleep quality was related to the behavioral performance observed in these tasks. To determine whether measures of sleep quality were related to overall performance, the average sleep duration $(400.3 \pm 61.8$, minutes) and sleep efficiency $(85.8 \% \pm$ $4.5 \%$ ) were computed over the three nights and compared to the average performance on each task. Average sleep duration and sleep efficiency adhered to normal distributions (all Shapiro-Wilk $p s>.05$ ) and were in the normal range of reported sleep parameters for healthy young adults (Ohayon, Carskadon, Guilleminault, \& Vitiello, 2004). After Bonferroni correction for multiple comparisons (adjusted alpha for the nine tasks, $p<.0055$ ), results indicated that there were no significant correlations between sleep duration or efficiency and the average task performance (i.e., averaged over the 10 sessions). Similarly, there were no significant correlations between these sleep measures and either the linear or quadratic coefficients of change for any of the tasks.

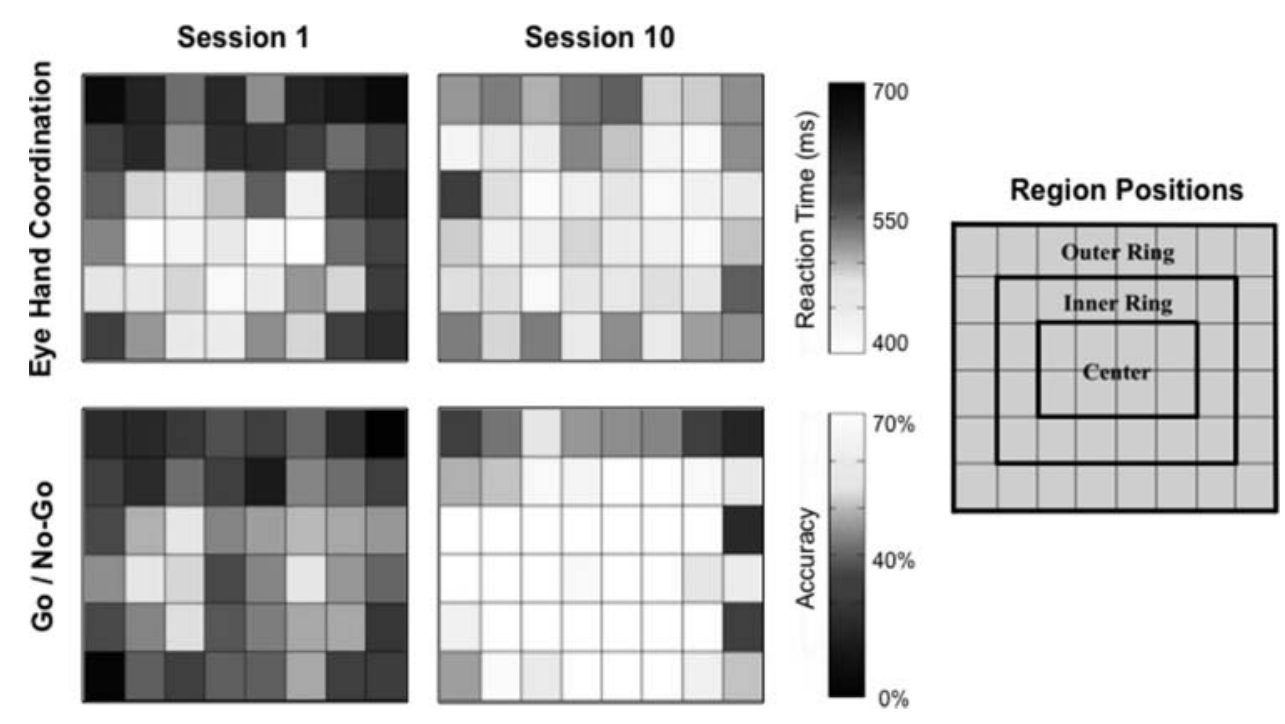

FIGURE 3. Heat maps of EHC response times and GNG accuracy rates. Note that lighter colors depict better performance. These maps indicate that improvements in performance for both tasks are widespread throughout the entire grid. 
TABLE 4. Reaction times in milliseconds for EHC and accuracy for GNG in each of the three regions of the display

\begin{tabular}{|c|c|c|c|c|c|c|}
\hline & \multicolumn{3}{|c|}{ Mean reaction times for $\mathrm{EHC}$} & \multicolumn{3}{|c|}{ Mean accuracy for GNG } \\
\hline & Center & Inner ring & Outer ring & Center & Inner ring & Outer ring \\
\hline Session 1 & 509.8 & 537.2 & 582.1 & $58 \%$ & $42 \%$ & $29 \%$ \\
\hline Session 10 & 445.0 & 458.7 & 507.1 & $78 \%$ & $72 \%$ & $49 \%$ \\
\hline
\end{tabular}

Note. Accuracy is computed as the ratio of hits versus possible targets (green dots) and excludes nontargets (red dots).

Since sleep has been suggested to aid in memory protection, rather than enhancement (Rickard et al., 2008), we also assessed whether the rate of learning differed for sessions performed within a day, versus between days. For this purpose the average differences between subsequent sessions on the same day (sessions 1-2, 2-3, 4-5, 5-6, 6-7, 8-9, 9-10) were compared to the average differences between subsequent sessions separated by a night (sessions $3-4,7-8$ ). A paired sample $t$ test showed no significant difference between withinday and between-day changes for each task ( $p s>.05)$.

\section{Discussion}

The Nike Sensory Station is a computerized psychometric battery that offers a quantitative platform for assessing a variety of sports-relevant sensorimotor abilities, ranging from low-level visual sensitivity to complex visuomotor coordination. In the present study we were interested in assessing how performance on these abilities changed as a result of repeated practice over a relatively short timeframe. By having participants practice this battery ten times over three days it was possible to quantify simple linear and quadratic coefficients of change to provide a preliminary mapping of learning for these skills. Using this approach, we found significant improvements in tasks with high visuomotor control demands and little change in tasks that primarily assessed visuoperceptual skills. In particular, substantial improvements in NFQ, PS, EHC, GNG, and RSP was observed, with some tasks improving as much as $60 \%$. Furthermore, the present findings demonstrated improvements in many different facets of the motor-based tasks such that different performance components within each task got better with practice. For example, both perceptual reaction speeds for detecting the target stimuli and the motor execution times for successful manual response significantly improved in the RSP task. Despite the initial hypotheses that the quality or duration of sleep would substantially impact the rate of learning and/or average performance, little evidence was found that sleep played a modulating role. In the following sections we discuss how these findings fit into the existing literature on perceptual and motor learning, speculate on why sleep did not impact behavior in the present study, and discuss these findings in the context of sports-specific sensorimotor assessment and training tools.

\section{Sensorimotor Learning}

Humans have the capacity to improve in many sensorimotor abilities in a relatively short period of time (Green \& Bavelier, 2008; Wolpert, Diedrichsen, \& Flanagan, 2011). Whether improving eye-hand coordination (Sailer, Flanagan, \& Johansson, 2005), arm reaching speed and accuracy (Cirstea, Ptito, \& Levin, 2006), or visual memory (Appelbaum, Cain, Schroeder, Darling, \& Mitroff, 2012; Klingberg, 2010) under the right conditions many visuoperceptual and visuomotor abilities can be improved through deliberate practice. Although improvements in low-leveled vision, such as visual acuity (Deveau et al., 2014; Poggio et al., 1992; Saarinen \& Levi, 1995) and contrast sensitivity (Li et al., 2009; Zhou et al., 2006), have been previously demonstrated under distinct testing conditions, investigations specific to test-retest performance on the Sensory Station, including our own, have predominately found motor learning, with little or no improvements in tasks assessing low-level vision (Erickson et al., 2011; Gilrein, 2014).

These findings are consistent with the idea that processes related to visual hardware, such as visual acuity, contrast sensitivity, and depth perception, are limited by the physical optometric properties of the visual system (Abernethy, 1986), which in the fovea are near optimal because of the high density of photoreceptors (Ciuffreda \& Wang, 2004). Although these skills may be improved through targeted training, there is little evidence showing that improvements will lead to better real-world performances (Abernethy \& Wood, 2001; Wood \& Abernethy, 1997). More often, elite performers are distinguished by their abilities to accurately perceive and react to relevant stimuli, known as visual software abilities, which are often influenced by the performer's prior experience and expectations (Abernethy, 1986). One exception in the present study is the improvements observed in performance on the NFQ task, which tests aspects of the visual hardware associated with accommodation and vergence. While accommodation and vergence are generally considered to be aspects of visual hardware, they are shown to be relatively malleable, as they can be compromised by traumatic brain injuries (Ciuffreda et al., 
2007) and improved through visual therapy (Yadav, Thiagarajan, \& Ciuffreda, 2014). It is also possible that the motor demands on eye movements needed to complete the NFQ task also led to compensatory motor improvements and enhanced performance (Abernethy, 1986).

The mechanisms by which the Sensory Station measures Static and DVA, CS, and DP tasks, which are all managed by a staircase procedure, could also partially explain the lack of improvements shown in these tasks. While the participants got to practice many dozen trails in each of these tasks over the 10 sessions, it is possible that the staircase procedure governing the stimulus displays may not have afforded adequate exposure at challenging enough thresholds to effectively improve low-level vision in a short period of time. As some studies have indicated that aspects of visual sensitivity can be improved with practice (Deveau et al., 2014; Li et al., 2009; Poggio et al., 1992; Saarinen \& Levi, 1995; Zhou et al., 2006), additional practice with these tasks may eventually yield significant practice effects.

Learning in sensorimotor skills has been of historical interest in psychology and researchers have long sought to characterize the time course of learning in these abilities. Typically, these studies have found that early performance on a novel task begins in a slow, effortful and error-prone manner until performance becomes more quick, accurate, and effortless through practice (Ackerman, 1987, 1988; Newell, 1991). As such, studies have often found practice effects to adhere closely to a power function (Anderson, 2000). The improvements seen in the current study, however, are primarily linear, with only EHC and GNG also showing slight, but significant, quadratic trends in overall task improvement. While higher order learning functions were not quantified in the current study, it is clear that the improvements here were primarily linear and, therefore, deviate somewhat from the expectation of power learning functions. One interpretation is that there is still more capacity for improvement on these tasks and that the ten practice sessions were not sufficient to capture the diminishing effects of practice seen in a typical power function. As such, additional practice may be expected to yield less dramatic improvements that eventually taper off and plateau. Furthermore, the dissimilar rates of improvements in the Sensory Station tasks indicate that the various visuomotor skills are developed at unique rates, so that additional practice may not yield uniform gains across tasks.

Last, it is important to consider the degree to which behavioral improvements might stem from visuomotor skill learning or from greater familiarity with the testing context and apparatus. Two aspects of the present results suggest that familiarity may not be strongly contribution to these findings. First, visuoperceptual abilities measured in the first four tasks did not improve, but were practiced the same amount as the tasks that did improve, indicating limited benefit of simple familiarity. Second, tasks like EHC and GNG that differ on only one dimension (added response inhibition), differ threefold in the amount of improvement due to practice. This difference would suggest that the greater learning in the GNG task might stem from relatively more improvements in response inhibition abilities, above visuomotor coordination alone, indicating specific task learning.

\section{Sleep, Psychomotor Performance, and Learning}

Over the past several years there has been growing evidence that sleep is important for the consolidation of certain types of memories (McGaugh, 2000; Stickgold, Hobson, \& Fosse, 2001). Studies addressing learning in a number of domains including sensory discrimination (Karni et al., 1994; Stickgold, James, \& Hobson, 2000), motor skills (Walker et al., 2002), and more general procedural-based skills (Peigneux, Laureys, Delbeuck, \& Maquet, 2001) have shown that sleep leads to greater improvements in performance, relative to the same period of wakefulness. Nonetheless, other studies have indicated that sleep may not be important for types of sensory and perceptual learning or may only occur once the perceptual system has been properly adapted to the stimuli (Aberg et al., 2009; Hussain et al., 2009), creating an open question as to the role of sleep in the learning of different skills.

In the present study, we did not find a significant relationship between performance and sleep. However, this study differed from many previous investigations in that the participants in this study were unconstrained in their sleep patterns. As indicated by the 400 average minutes of sleep, these individuals were well within the range of normal uninterrupted sleep for a college-ages sample (Ohayon et al., 2004). As such, sleep may not have been sufficiently deprived as to interact with learning or general performance. The fact that learning did not decrease over the interval between successive testing days may support the notions that sleep acts to assist in the stabilization of memories (Rickard et al., 2008). Further studies perturbing normal sleep schedules will help to identify the role of sleep in performance on this battery.

\section{Conclusions and Implications}

The present study demonstrated differences in capacity and rate of learning across perceptual and motor domains in a sample of healthy young adults. These findings have relevance to the realm of skill acquisition that has sought to better understand the human capacity for learning and the factors that contribute to these abilities (Macnamara et al., 2014). Further, these results may have implications toward the utility of the Nike Sensory Station for athletic assessment and training approaches. For example, there is a long history of literature demonstrating that superior sensorimotor abilities differ for more or less accomplished individuals in numerous occupational pursuits, such as in sports (Mann 
et al., 2007; Poltavski \& Biberdorff, 2014; Voss et al., 2010), medicine (Datta et al., 2002), and military activities (King et al., 2013). Moreover, recent demonstrations that specific abilities assessed by the Sensory Stations are predictive of greater on-field performance (Poltavski \& Biberdorff, 2014) and injury avoidance (Harpham et al., 2014) indicate that training of these skills may lead to improved outcomes in athletic and other real-world pursuits. As such the current findings point towards specific abilities that may show the greatest learning and therefore can be used to guide interventions aimed at improving success in these important applications. Future research may build on these findings to assess the transfer of learning on this sensorimotor battery to on-field performance, and other real-world pursuits.

\section{Acknowledgments}

Thanks to Lauren Hughes, Tarik Bel-Bahar, Annie Apple, Floyd Wilks Jr., Clara Colombatto, Yvonne Lu, Gabriela Asturas, and Eliza Gentzler for assistance with data collection; to the Duke Sleep Clinic for their guidance in actigraphy collection and analysis; and to the Duke Visual Cognition Lab for their aid in experiment setup. During the execution of this research project, Stephen R. Mitroff served on an advisory board for Nike Inc., the producer of the Sensory Station.

\section{Funding}

This research was funded by grant support to L. Gregory Appelbaum and Stephen R. Mitroff through DARPA grant \# D12AP00025-002.

\section{References}

Aberg, K. C., Tartaglia, E. M., \& Herzog, M. H. (2009). Perceptual learning with Chevrons requires a minimal number of trials, transfers to untrained directions, but does not require sleep. Vision Research, 49, 2087-2094. doi:10.1016/j.visres.2009.05.020

Abernethy, B. (1986). Enhancing sports performance through clinical and experimental optometry. Clinical \& Experimental Optometry, 69, 189-196.

Abernethy, B., \& Wood, J. M. (2001). Do generalized visual training programmes for sport really work? An experimental investigation. Journal of Sports Sciences, 19, 203-222. doi:10.1080/ 026404101750095376

Ackerman, P. (1987). Individual differences in skill learning: An integration of psychometric and information processing perspectives. Psychological Bulletin, 102, 3-27.

Ackerman, P. (1988). Determinants of indvidual differences during skill acquistion: Cognitive abilities and information processing. Journal of Experimental Psychology, 117, 288-318.

Anderson, J. R. (2000). Learning and memory. New York, NY: Wiley.

Appelbaum, L. G., Cain, M. S., Schroeder, J. E., Darling, E. F., \& Mitroff, S. R. (2012). Stroboscopic visual training improves information encoding in short-term memory. Attention, Percep- tion and Psychophysics, 74, 1681-1691. doi:10.3758/s13414012-0344-6

Appelbaum, L. G., Schroeder, J. E., Cain, M. S., \& Mitroff, S. R. (2011). Improved visual cognition through stroboscopic training. Frontiers Psychology, 2, 276.

Ball, K., \& Sekuler, R. (1982). A specific and enduring improvement in visual motion discrimination. Science, 218, 697-698.

Carney, C. E., Buysse, D. J., Ancoli-Israel, S., Edinger, J. D., Krystal, A. D., Lichstein, K. L., \& Morin, C. M. (2012). The consensus sleep diary: Standardizing prospective sleep selfmonitoring. Sleep, 35, 287-302. doi:10.5665/sleep.1642

Cirstea, C. M., Ptito, A., \& Levin, M. F. (2006). Feedback and cognition in arm motor skill reacquisition after stroke. Stroke, 37, 1237-1242. doi:10.1161/01.STR.0000217417.89347.63

Ciuffreda, K. J., Kapoor, N., Rutner, D., Suchoff, I. B., Han, M. E., \& Craig, S. (2007). Occurrence of oculomotor dysfunctions in acquired brain injury: A retrospective analysis. Optometry, 78, 155-161. doi:10.1016/j.optm.2006.11.011

Ciuffreda, K. J., \& Wang, B. (2004). Vision training and sports. In G. K. Hung (Ed.), Biomedical engineering principles in sports (pp. 407-433). New York, NY: Kluwer Academic / Plenum Publishers.

Datta, V., Chang, A., Mackay, S., \& Darzi, A. (2002). The relationship between motion analysis and surgical technical assessments. American Journal of Surgery, 184, 70-73.

Deveau, J., Lovcik, G., \& Seitz, A. R. (2014). Broad-based visual benefits from training with an integrated perceptual-learning video game. Vision Research, 99, 134-140.

Erickson, G. B. (2007). Sports vision: Vision care for the enhancement of sports performance. Oxford, England: ButterworthHeinemann.

Erickson, G. B., Citek, K., Cove, M., Wilczek, J., Linster, C., Bjarnason, B., \& Langemo, N. (2011). Reliability of a computerbased system for measuring visual performance skills. Optometry, 82, 528-542. doi:10.1016/j.optm.2011.01.012

Frank, M. G., \& Benington, J. H. (2006). The role of sleep in memory consolidation and brain plasticity: Dream or reality? Neuroscientist, 12, 477-488. doi:10.1177/1073858406293552

Gilrein, T. (2014). Reliable change indices of visual and sensory performance measures. Master's thesis, University of North Carolina, Chapel Hill.

Green, C. S., \& Bavelier, D. (2008). Exercising your brain: A review of human brain plasticity and training-induced learning. Psychology of Aging, 23, 692-701. doi:10.1037/a0014345

Griffin, G. R., \& Koonce, J. M. (1996). Review of psychomotor skills in pilot selection research of the U.S. military services. International Journal of Aviation Psychology, 6, 125-147. doi:10.1207/s15327108ijap0602_2

Harpham, J. A., Mihalik, J. P., Littleton, A. C., Frank, B. S., \& Guskiewicz, K. M. (2014). The effect of visual and sensory performance on head impact biomechanics in college football players. Annals of Biomedical Engineering, 42, 1-10. doi:10.1007/ s10439-013-0881-8

Hitzeman, S. A., \& Beckerman, S. A. (1993). What the literature says about sports vision. Optom Clin, 3, 145-169.

Hussain, Z., Sekuler, A. B., \& Bennett, P. J. (2009). How much practice is needed to produce perceptual learning? Vision Research, 49, 2624-2634. doi:10.1016/j. visres.2009.08.022

Karni, A., Meyer, G., Rey-Hipolito, C., Jezzard, P., Adams, M., Turner, R., \& Underleider, L. G. (1998). The aquisition of skilled motor performance: Fast and slow exerience-driven changes in primary motor cortex. Proceedings of the National Academy of Sciences of the USA, 95, 861-868. 
Karni, A., Tanne, D., Rubenstein, B. S., Askenasy, J. J., \& Sagi, D. (1994). Dependence on REM sleep of overnight improvement of a perceptual skill. Science, 265, 679-682.

King, R., Carretta, T., Retzlaff, P., Barton, E., Ree, M. J., \& Teachout, M. (2013). Standard cognitive psychological tests predict military pilot training outcome. Aviation Psychology and Applied Human Factors, 3, 28-38.

Klingberg, T. (2010). Training and plasticity of working memory. Trends in Cognitive Sciences, 14, 317-324.

Lesmes, L. A., Lu, Z. L., Baek, J., \& Albright, T. D. (2010). Bayesian adaptive estimation of the contrast sensitivity function: The quick CSF method. Journal of Vision, 10(3), 17.1-21. doi:10.1167/10.3.17

Li, R., Polat, U., Makous, W., \& Bavelier, D. (2009). Enhancing the contrast sensitivity function through action video game training. Nature Neuroscience, 12, 549-551.

Maan, Z. N., Maan, I. N., Darzi, A. W., \& Aggarwal, R. (2012). Systematic review of predictors of surgical performance. British Journal of Surgery, 99, 1610-1621. doi:10.1002/bjs.8893

Macnamara, B. N., Hambrick, D. Z., \& Oswald, F. L. (2014). Deliberate practice and performance in music, games, sports, education, and professions: A meta-analysis. Psychological Science, 25, 1608-1618. doi:10.1177/0956797614535810

Mann, D. T., Williams, A. M., Ward, P., \& Janelle, C. M. (2007). Perceptual-cognitive expertise in sport: A meta-analysis. Journal of Sport and Exercise Psychology, 29, 457-478.

McGaugh, J. L. (2000). Memory-a century of consolidation. Science, 287, 248-251.

Newell, K. M. (1991). Motor skill acquisition. Annual Review of Psychology, 42, 213-237. doi:10.1146/annurev.ps.42.020191.001241

Ohayon, M. M., Carskadon, M. A., Guilleminault, C., \& Vitiello, M. V. (2004). Meta-analysis of quantitative sleep parameters from childhood to old age in healthy individuals: Developing normative sleep values across the human lifespan. Sleep, 27, $1255-1273$.

Peigneux, P., Laureys, S., Delbeuck, X., \& Maquet, P. (2001). Sleeping brain, learning brain. The role of sleep for memory systems. Neuroreport, 12, A111-A124.

Poggio, T., Fahle, M., \& Edelman, S. (1992). Fast perceptual learning in visual hyperacuity. Science, 256, 1018-1021.

Poltavski, D., \& Biberdorff, D. (2015). The role of visual perception measures used in sports vision programs in predicting actual game performance in Division I collegiate hockey players. Journal of Sports Science. 33(6), 597-608.

Rickard, T. C., Cai, D. J., Rieth, C. A., Jones, J., \& Ard, M. C. (2008). Sleep does not enhance motor sequence learning. Journal of Experimental Psychology: Learning Memory and Cognition, 34, 834-842. doi:10.1037/0278-7393.34.4.834

Saarinen, J., \& Levi, D. M. (1995). Perceptual learning in vernier acuity: What is learned? Vision Research, 35, 519-527.

Sailer, U., Flanagan, J. R., \& Johansson, R. S. (2005). Eye-hand coordination during learning of a novel visuomotor task.
Journal of Neuroscience, 25, 8833-8842. doi:10.1523/JNEUROSCI.2658-05.2005

Shiu, L., \& Pashler, H. (1992). Improvement in line orientation discrimination is retinally local but dependent on cognitive set. Perception \& Psychophysics, 52, 582-588.

Stickgold, R., Hobson, J. A., \& Fosse, M. (2001). Sleep, learning, and dreams: Off-line memory reprocessing. Science, 294, 1052-1057.

Stickgold, R., James, L., \& Hobson, J. A. (2000). Visual discrimination learning requires sleep after training. Nature Neuroscience, 3, 1237-1238.

Vogels, R., \& Orban, G. A. (1985). The effect of practice on the oblique effect in line orientation judgements. Vision Research, $25,1679-1687$.

Voss, M. W., Kramer, A. F., Basak, C., Parkash, R. S., \& Roberts, B. (2010). Are expert athletes expert in the cognitive laboratory? A meta-analytic review of cognition and sport expertise. Applied Cognitive Psychology, 24, 812-826.

Walker, M. P., Brakefield, T., Morgan, A., Hobson, J. A., \& Stickgold, R. (2002). Practice with sleep makes perfect: Sleepdependent motor skill learning. Neuron, 35, 205-211.

Walker, M. P., \& Stickgold, R. (2006). Sleep, memory, and plasticity. Annual Review of Psychology, 57, 139-166. doi:10.1146/ annurev.psych.56.091103.070307

Wang, L., Bel-Bahar, T., Hughes, L., Krasich, K., Mitroff, S. R., \& Appelbaum, L. G. (2015). Mapping the structure of perceptual and visual-motor abilities in healthy young adults. Acta Psychologica, 157, 74-84.

Willis, S., Tennstedt, S., Marsiske, M., Ball, K., Elias, J., Koepke, K., \& Wright, E. (2006). Long-term effects of cognitive training on everyday functional outcomes in older adults. Journal of the American Medical Association, 296, 2805-2814.

Wolpert, D. M., Diedrichsen, J., \& Flanagan, J. R. (2011). Principles of sensorimotor learning. Nature Reviews Neuroscience, 12, 739-751. doi:10.1038/nrn3112

Wood, J. M., \& Abernethy, B. (1997). An assessment of the efficacy of sports vision training programs. Optometry and Vision Science, 74, 646-659.

Yadav, N. K., Thiagarajan, P., \& Ciuffreda, K. J. (2014). Effect of oculomotor vision rehabilitation on the visual-evoked potential and visual attention in mild traumatic brain injury. Brain Injury, 28, 922-929. doi:10.3109/02699052.2014.887227

Yoo, H., Reichow, A. W., \& Erickson, G. (2011, September). Stereoacuity of athletes in primary and non-primary gazes. Paper presented at the Vision Science Society Annual Meeting, Naples, FL.

Zhou, Y., Huang, C., Xu, P., Tao, L., Qiu, Z., Li, X., \& Lu, Z. (2006). Perceptual learning improves contrast sensitivity and visual acuity in adults with anisometropic amblyopia. Vision Research, 46, 739-750.

Received May 26, 2015

Revised September 24, 2015

Accepted October 23, 2015 